\title{
Frequency of nonaspirin NSAID-relevant coexisting medical conditions in the primary-care setting: a retrospective database review
}

This article was published in the following Dove Medical Press journal:

Therapeutics and Clinical Risk Management

Leslie Bloom'

Kathleen E Boyle ${ }^{2}$

Andrew E Myers'

Claire Blacketer ${ }^{3}$

Rachel Weinstein ${ }^{3}$

'Johnson \& Johnson Consumer Inc, McNeil Consumer Healthcare Division, Fort Washington, PA, USA;

${ }^{2} \mathrm{KE}$ Boyle Consultants, Exton, PA, USA; ${ }^{3}$ Janssen Research and Development, Titusville, NJ, USA
Correspondence: Leslie Bloom Johnson \& Johnson Consumer, McNeil Consumer Healthcare Division, 7050 Camp Hill Road, Fort Washington, PA 19034, USA

Tel + I 2152738729

Fax +I 2152734232

Email Ibloom@its.jnj.com
Background: Coexisting medical conditions and concomitant medications contribute to treatment challenges primary-care professionals (PCPs) face daily. The current study assessed the extent and distribution of nonaspirin NSAID-relevant coexisting medical conditions of interest (CMCOI) in patients visiting PCPs.

Methods: This retrospective database review analyzed data from three large health-care claim databases to identify the frequency of nonaspirin NSAID-relevant CMCOI among adults aged $\geq 18$ years with a PCP visit in 2013. Claim databases employed were the Truven Health MarketScan ${ }^{\circledR}$ Commercial Claims and Encounters database, representative of the privately insured (PI) population; Truven Health MarketScan Multi-State Medicaid, representative of the Medicaid population (Medicaid); and Truven MarketScan Medicare Supplemental, representative of the Medicare population with employer-based supplemental Medicare insurance (Medicare-Supplement). Nonaspirin NSAID-relevant CMCOI, asthma, cardiovascular risk factors, gastrointestinal bleeding risk factors, and renal insufficiency were chosen based on US NSAID over-the-counter Drug Facts label warnings. Frequency of CMCOI was determined for those without and with a musculoskeletal diagnosis.

Results: In each database, $\geq 19 \%$ (19.0\% PI, 29.9\% Medicaid, 33.6\% Medicare-Supplement) had a musculoskeletal diagnosis. A greater proportion of individuals with a musculoskeletal diagnosis had one or more CMCOI compared with those without a musculoskeletal diagnosis (61.3\% vs 50.4\% PI, 78.1\% vs $66.8 \%$ Medicaid, $87.1 \%$ vs $82.3 \%$ Medicare-Supplement). The frequency of one or more CMCOI increased with age in each database. Across databases among CMCOI, cardiovascular risk factors were most common, followed by gastrointestinal bleeding risk factors, and proportions were higher among those with a musculoskeletal diagnosis.

Conclusion: These data confirm the high frequency of nonaspirin NSAID-relevant CMCOI among patients presenting to PCPs for musculoskeletal diagnosis, as well as among older patients. These analyses reinforce the critical role health-care professionals can play in identifying patients with nonaspirin NSAID-relevant CMCOI, providing those patients with ongoing guidance on appropriate choice and use of over-the-counter analgesics, and educating patients about the impact aging, health status, concomitant conditions, and medicines have on selection of all medicines, including analgesics.

Keywords: coexisting medical conditions, NSAIDs, musculoskeletal disease, health claim databases, OTC analgesics

\section{Plain-language summary}

Prescription or over-the-counter (OTC) pain relievers, such as NSAIDs, are often prescribed or recommended during patient visits. Using data from three large US health-care insuranceclaim databases, we calculated the percentage of adults having a chronic medical condition 
(ie, asthma, heart conditions, gastrointestinal bleeding risk, or kidney disease) that could put them at risk of a side effect when taking an OTC NSAID. The data showed that at least $50 \%$ of patients had one or more of these underlying medical conditions and heart conditions were generally the most common. The percentage of patients with these underlying conditions increased with age and was higher among those with a musculoskeletal diagnosis. Women were as likely as men to have these conditions. When making recommendations, health-care professionals can reduce their patients' risk of experiencing reactions to drugs by reminding patients that instructions on OTC medicines (ie, Drug Facts label) are not suggestions, but rather are specific instructions to follow closely. As patients age and their medical conditions change, different warnings on Drug Facts labels may apply to them. They can contact their health-care professional with questions or visit the Get Relief Responsibly (www.getreliefresponsibly.com) or Alliance for Rational Use of NSAIDS (www.nsaidalliance.com) websites to find information and tools to choose, use, store, and dispose of nonprescription medicines safely.

\section{Introduction}

In the US, people visit their primary health-care professional (HCP) on average three times annually. While the most common reason for an HCP visit is preventive (19.1\% of visits), the second-most common reason is musculoskeletal and connective tissue diseases (10.1\%). ${ }^{1}$ These patients are among those most likely to be prescribed or recommended to use analgesics.

In 2015, the National Ambulatory Medical Care Survey found that patients had one or more chronic conditions at $61.0 \%$ of office visits: $24.6 \%$ of the visits related to one chronic condition, $14.6 \%$ to two, and $21.8 \%$ to more than three. ${ }^{1}$ The National Health Interview Survey, which provides patientreported health information, identified arthritis $(21.6 \%)$, chronic joint symptoms (28.3\%), diabetes (8.8\%), gastrointestinal ulcers $(5.7 \%)$, and kidney disease $(1.9 \%)$ as among the most common chronic diseases with which Americans report being diagnosed. ${ }^{2}$ Considering these data, it is not surprising that patients in the US discussed medication(s) with their HCP at $76 \%$ of all office visits. Analgesics, including narcotics, nonnarcotics, and NSAIDs, were the most commonly discussed medications, accounting for $11.0 \%$ of all drug mentions. ${ }^{1}$

Research, evidence-based guidelines, and recommendations from professional societies and regulatory bodies identify that when recommending a treatment plan, which may include either prescription or over-the-counter (OTC) analgesics, HCPs should consider patient risks, ie, the patient's age, concomitant medications, and coexisting medical conditions, such as asthma, cardiovascular disorders, gastrointestinal bleeding, renal insufficiency, and liver disease. ${ }^{3-6}$ Drug Facts labels for OTC NSAIDs contain clear warnings describing who should not use the medication and who should seek doctor or pharmacist advice before using the product. Notably, in April 2009 the FDA published updated organ-specific warnings, including stomach-bleeding warnings, for OTC NSAID labels in the Federal Register to be implemented within 12 months. ${ }^{7}$ Again, in 2016 OTC NSAID new-drug-application holders received FDA correspondence directing them to update nonaspirin OTC NSAID labels to include warnings that alerted patients with cardiovascular conditions to speak with their doctor before using. These label changes were approved for implementation in 2017. When recommending an analgesic, HCPs can take the opportunity to review an OTC Drug Facts label with patients and explain why specific medicines and not other medicines are recommended based on the patient's medical history and current medications. HCPs should educate patients that medicines are prescribed or recommended based on the patient's current health status and that previous prescriptions or recommendations may no longer be appropriate, due to changes in the patient's age, medications, or medical conditions.

Information from health-care claim databases that are representative of the majority of Americans can provide insights into the nature of the population receiving NSAIDS and thereby provide important reminders to clinicians to consider comorbidities when prescribing. Therefore, three health-claim databases were analyzed to perform a descriptive analysis of the extent and distribution of nonaspirin NSAID-relevant coexisting medical conditions in patients with and without a musculoskeletal disorder who had visited a primary-care professional (PCP).

\section{Methods}

A retrospective database review was performed.

\section{Data sources}

Three large health-care claim databases were the sources of patient-level information: the Truven Health MarketScan ${ }^{\circledR}$ Commercial Claims and Encounters database, representative of the PI population; Truven Health MarketScan MultiState Medicaid (Medicaid), representative of the Medicaid population; and Truven MarketScan Medicare Supplemental (Medicare-Supplement), representative of the Medicare population with employer-based supplemental Medicare insurance.

All three databases capture diagnoses and procedures submitted for office visits and hospitalizations and prescription-drug dispensing in retail and mail-order pharmacies. Patient-level information in all MarketScan database records is deidentified and fully compliant with US patientconfidentiality requirements, including the Health Insurance 
Portability and Accountability Act (HIPAA) of 1996. Further, Truven databases are certified to satisfy conditions described in sections 164.514 (a)-(b)1ii of the HIPAA privacy rule regarding determination and documentation of statistically deidentified data. A description of the data available in each of the three databases can be found in the Supplementary material.

\section{Selection and definition of relevant coexisting medical conditions}

Nonaspirin NSAID-relevant coexisting medical conditions of interest (CMCOI) were selected based on review of US OTC NSAID labels and consideration of which NSAID-relevant coexisting medical condition(s) are most frequently encountered in typical PCP populations based on other literature. ${ }^{1,2,8}$ Based on these considerations, asthma, cardiovascular risk, gastrointestinal bleeding risk, and renal impairment (including renal failure, hereafter referred to as renal impairment) were chosen for analysis. Hepatic cirrhosis was not chosen as a CMCOI, due to its lower frequency in the primary-care setting compared to the selected CMCOI. ${ }^{8}$ Throughout this report, CMCOI relate to the four specific conditions evaluated, and do not indicate the absence or presence of other medical conditions.

Medical concepts associated with the CMCOI were defined by the authors. Specifically, one or more standard Systematized Nomenclature of Medicine (SNOMED) concepts (Observational Medical Outcomes Partnership Vocabulary version 5.0, April 3, 2015) were used for each of the four CMCOI, and all terms from the lists were reviewed to exclude terms not consistent with the concept of interest. For example, for gastrointestinal bleeding risk factors, all descendant terms (using the SNOMED hierarchy) of the SNOMED concepts for gastrointestinal hemorrhage and gastrointestinal ulcer were used. In addition, gastrointestinal bleeding risk factors also included all drugs linked to the RxNorm concepts for anticoagulants and corticosteroids (Table 1). All diagnosis and drug-code lists used in this study are available from the authors.

The New England Institutional Review Board (NEIRB, 12-284) determined that studies conducted in these databases did not meet the definition of human-subject research. Therefore, board review and approval of the protocol was not required.

\section{Sample selection and study-population characteristics}

Patients aged $\geq 18$ years in 2013 with a visit to a PCP in the same year were included in the study. Only patients
Table I Medical concept(s) used to identify patients with NSAIDrelevant coexisting medical conditions of interest

\begin{tabular}{|l|l|}
\hline Medical condition & Medical concepts \\
\hline Asthma & Asthma \\
\hline Cardiovascular risks & $\begin{array}{l}\text { Acute myocardial infarction, cerebrovascular } \\
\text { disease, disorder of coronary artery, heart } \\
\text { failure, hypertensive disorder }\end{array}$ \\
\hline $\begin{array}{l}\text { Gastrointestinal } \\
\text { bleeding risks }\end{array}$ & $\begin{array}{l}\text { Gastrointestinal hemorrhage, gastrointestinal } \\
\text { ulcer, anticoagulant, alcohol dependence }\end{array}$ \\
\hline Renal impairment & Renal impairment \\
\hline
\end{tabular}

with $\geq 1$ year of enrollment prior to their first PCP visit in 2013 were included, so that medical history was available to review. For this analysis, PCP specialties were defined as family practice, geriatric medicine, or internal medicine.

Patients' electronic claim records were examined to identify the presence or absence of a musculoskeletal diagnosis and the index date. To identify the musculoskeletal diagnosis population within each database, the first visit with a musculoskeletal complaint in the primary-care outpatient setting in 2013 was considered the index date. Among those without a musculoskeletal complaint within the year, the first visit in the primary-care outpatient setting was the index date. Once each study population had been identified, each patient's electronic claim records were examined for evidence of a CMCOI in the 365 days prior to and including the index date. Patients were then grouped together based on their age, sex, and number and type of CMCOI or lack thereof.

\section{Statistical analyses}

All patient characteristics are described using means and SD for continuous variables and frequencies and percentages for categorical variables.

\section{Results}

Individuals within the PI database ranged between ages of 18 and 74 years; those between 65 and 74 years likely represent individuals who maintain private insurance while they are enrolled in Medicare. The Medicaid database includes individuals aged from 18 to $>85$ years. The Medicare-Supplement database includes only individuals with both a Medicare-Supplemental health-care plan and Medicare benefits. Table 2 identifies characteristics of individuals enrolled in each database. Although a few individuals aged 18-64 years were identified in the MedicareSupplement database, only individuals aged $>65$ years were included in all subsequent analyses. 


\section{Overall patient characteristics by database}

Mean ages among patients in the overall population and in the musculoskeletal diagnosis population in the PI and Medicaid populations were similar. In both the PI and Medicaid population databases, the percentage of individuals with a musculoskeletal diagnosis increased with increasing age up to age 65 years. In the Medicaid population, the percentage of individuals with a musculoskeletal diagnosis decreased with age $>65$ years (Table 2 ).

\section{Prevalence of one or more CMCOI by database}

Half $(50.4 \%)$ the patients in the PI database had one or more CMCOI. In both the Medicaid (66.8\%) and MedicareSupplement $(82.3 \%)$ populations, a greater proportion of patients had one or more CMCOI (Table 2). The MedicareSupplement population had the highest proportion of patients with one $(45.9 \%)$, two $(28.8 \%)$, three $(7.1 \%)$, or four $(0.6 \%)$ CMCOI. In comparison, the PI database had the lowest proportion of patients with one (37.6\%), two (11.3\%), three
(1.5\%), or four $(0.1 \%)$ CMCOI. While $61.3 \%$ of patients with a musculoskeletal diagnosis in the PI population had been diagnosed with one or more CMCOI, $78.1 \%$ of patients in the Medicaid and $87.1 \%$ in the Medicare-Supplement populations with a musculoskeletal diagnosis had been diagnosed with one or more of these conditions (Table 2, Figure 1).

The proportion of patients with a musculoskeletal diagnosis and one or more CMCOI increased with increasing age within each database. Furthermore, the percentage of individuals within each age-group with one or more $\mathrm{CMCOI}$ was higher among those with a musculoskeletal diagnosis compared to the overall population (Figure 2).

\section{Prevalence of specific CMCOI by database}

Cardiovascular risk factors were generally the most commonly identified CMCOI across the three databases (Figure 3). Cardiovascular risk factors were lower among females than males within each database (PI, 26.2\% and $34.2 \%$, respectively; Medicaid, $50.0 \%$ and $55.5 \%$, respectively; Medicare-Supplement, $70.3 \%$ and $74.1 \%$, respectively).

Table 2 Demographic characteristics of individuals in the Privately Insured, Medicaid, and Medicare-Supplement databases by total population within database and by musculoskeletal diagnosis

\begin{tabular}{|c|c|c|c|c|c|c|}
\hline \multirow{2}{*}{$\begin{array}{l}\text { Patients with PCP } \\
\text { visits in } 2013^{a}\end{array}$} & \multicolumn{3}{|c|}{ Total population ${ }^{b}$} & \multicolumn{3}{|c|}{ Musculoskeletal diagnosis ${ }^{c}$} \\
\hline & $\begin{array}{l}\text { Privately } \\
\text { Insured }\end{array}$ & Medicaide $^{\mathrm{e}}$ & $\begin{array}{l}\text { Medicare- } \\
\text { Supplement }\end{array}$ & $\begin{array}{l}\text { Privately } \\
\text { Insured }\end{array}$ & Medicaide $^{\mathrm{e}}$ & $\begin{array}{l}\text { Medicare- } \\
\text { Supplement }\end{array}$ \\
\hline$n$ & $2,967,442$ & 584,787 & 377,079 & $565,07 \mid$ & $|74,84|$ & 126,595 \\
\hline$\%$ Total population & 100.0 & 100.0 & 100.0 & 100.0 & 100.0 & 100.0 \\
\hline Age, mean (SD), years & $45.3(13.0)$ & $49.4(20.0)$ & $76.3(7.7)$ & $48.2(12.3)$ & $53.8(18.6)$ & $76.8(7.8)$ \\
\hline$\%>60$ years old & 14.9 & 31.6 & 99.6 & 19.7 & 37.1 & 99.6 \\
\hline \multicolumn{7}{|l|}{ Age group, years, $\%$} \\
\hline $18-44$ & 43.1 & 42.8 & 0.0 & 32.8 & 31.5 & 0.0 \\
\hline $45-64$ & 55.3 & 33.3 & 1.2 & 65.5 & 40.8 & 1.4 \\
\hline $65-84$ & 1.6 & 18.6 & 82.0 & 1.7 & 21.2 & 80.2 \\
\hline $85+$ & 0.0 & 5.3 & 16.7 & 0.0 & 6.5 & 18.4 \\
\hline \multicolumn{7}{|l|}{ Sex, \% } \\
\hline Female & 57.3 & 68.7 & 56.0 & 58.3 & 71.0 & 62.0 \\
\hline \multicolumn{7}{|c|}{ Coexisting medical condition(s), \% } \\
\hline One or more & 50.4 & 66.8 & 82.3 & 61.3 & 78.1 & 87.1 \\
\hline Asthma & 5.8 & 12.5 & 5.9 & 7.3 & 16.1 & 7.6 \\
\hline Cardiovascular & 29.7 & 51.7 & 72.0 & 34.3 & 61.8 & 74.6 \\
\hline Gastrointestinal & 28.0 & 26.7 & 37.4 & 42.1 & 38.8 & 50.4 \\
\hline Renal & 1.4 & 9.3 & 11.7 & 2.0 & 10.8 & 13.6 \\
\hline
\end{tabular}

Notes: Values are $\mathrm{n}$ (percentage) unless otherwise indicated. Comorbidities were measured in the 365 days prior to and including the index date. Demographics were measured as of the index date. ${ }^{\mathrm{A}} \mathrm{A}$ patient could have more than one comorbidity. The code occurred up to 365 days prior to the index date through and including the index date. ' ${ }^{\text {Total }}$ population: individuals who had a visit with the PCP in 2013. CMusculoskeletal diagnosis: individuals whose medical claim record reflected a musculoskeletal diagnosis code on the index date and at least one time in the preceding 365 days. ${ }^{d}$ Privately Insured: Truven Health MarketScan ${ }^{\circledR}$ Commercial Claims and Encounters database. eMedicaid: Truven Health MarketScan Multi-State Medicaid. 'Medicare Supplement: Truven MarketScan Medicare-Supplemental.

Abbreviation: PCP, primary-care professional. 


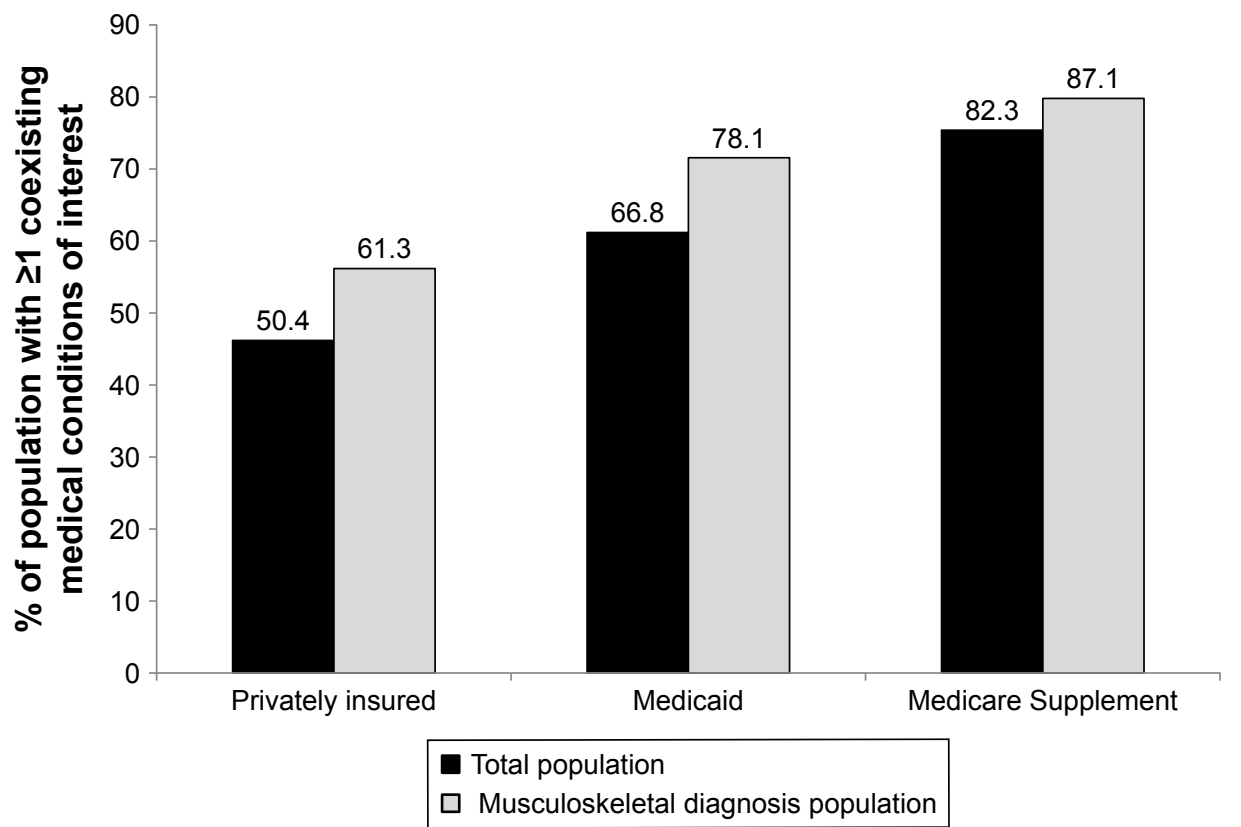

Figure I Percentage of total population and MSD population in each database with one or more coexisting medical conditions of interest.

Notes: Privately Insured: Truven Health MarketScan ${ }^{\circledR}$ Commercial Claims and Encounters database. Medicaid: Truven Health MarketScan Multi-State Medicaid. Medicare Supplement: Truven MarketScan Medicare-Supplemental.

Abbreviation: MSD, musculoskeletal diagnosis.

Similar percentages of individuals in the PI (28.0\%) and Medicaid (26.7\%) databases had gastrointestinal bleeding risk factors, and more individuals in the Medicare-Supplement database (37.4\%) had such risk factors (Figure 3). Similar percentages of women and men in each database were diagnosed with a gastrointestinal bleeding risk factor. Asthma was diagnosed twice as frequently in the Medicaid (12.5\%) as the PI (5.8\%) and Medicare-Supplement (5.9\%) databases (Figure 3).

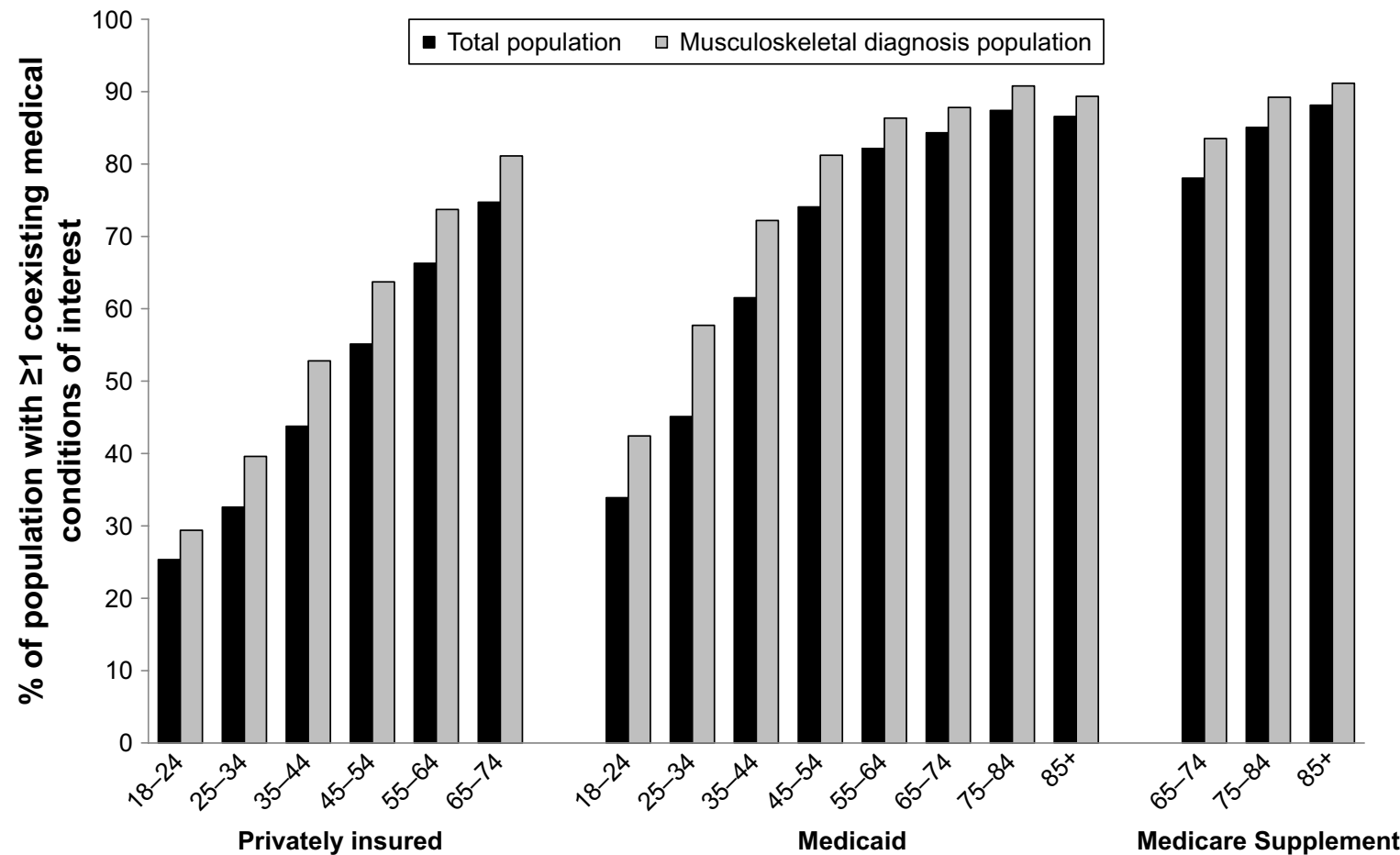

Age (years)

Figure 2 Percentage of population with one or more coexisting medical condition of interest by age-group, total, and MSD populations.

Notes: Privately Insured: Truven Health MarketScan ${ }^{\circledR}$ Commercial Claims and Encounters database. Medicaid: Truven Health MarketScan Multi-State Medicaid. Medicare Supplement: Truven MarketScan Medicare-Supplemental.

Abbreviation: MSD, musculoskeletal diagnosis. 


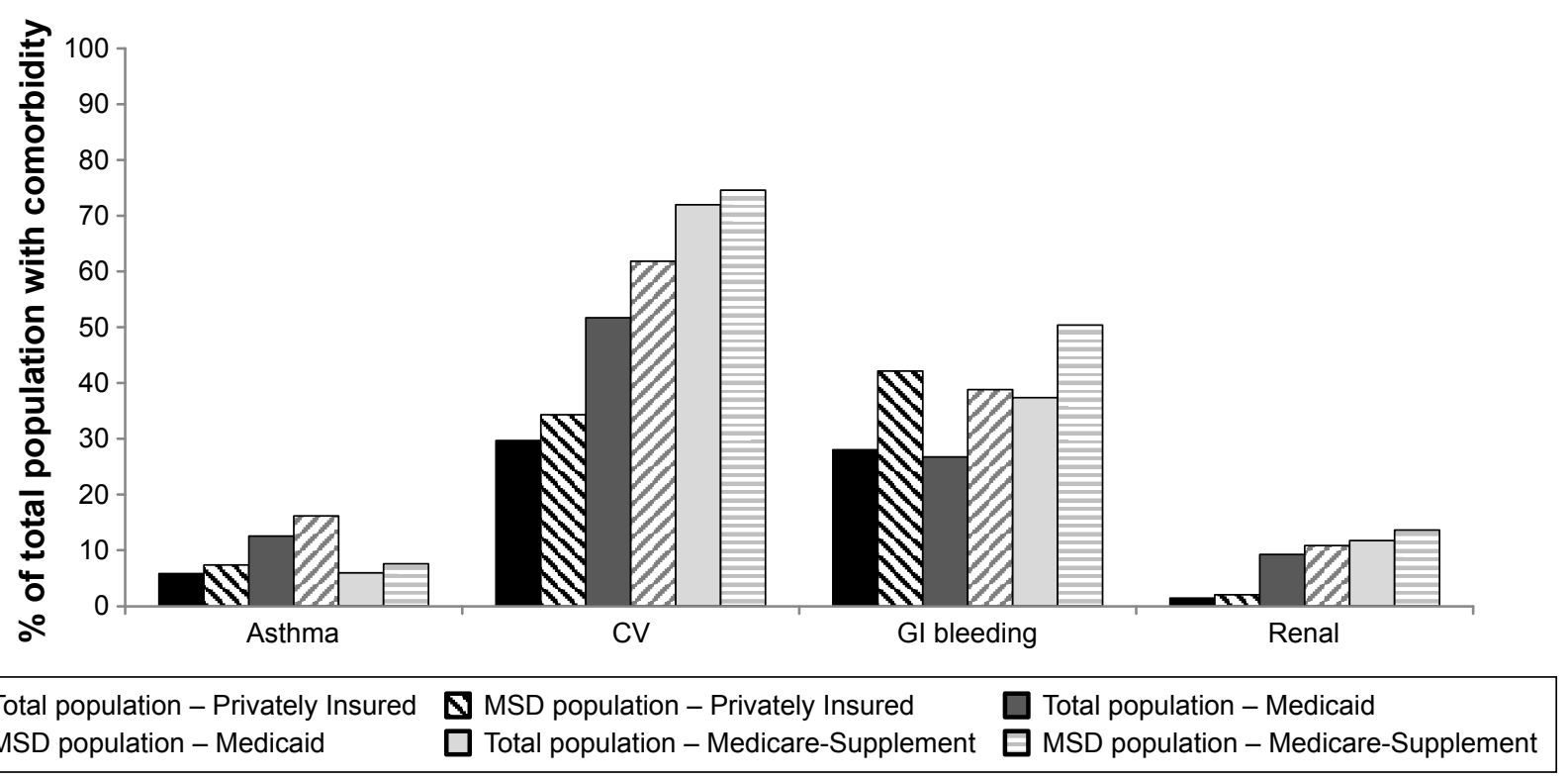

Figure 3 Percentage of total population and MSD population in each database with specific coexisting medical condition of interest.

Notes: Privately Insured: Truven Health MarketScan ${ }^{\circledR}$ Commercial Claims and Encounters database. Medicaid: Truven Health MarketScan Multi-State Medicaid. Medicare Supplement: Truven MarketScan Medicare-Supplemental.

Abbreviations: CV, cardiovascular; GI, gastrointestinal; MSD, musculoskeletal diagnosis.

Overall, the prevalence of renal disease was low in all three databases relative to cardiovascular and gastrointestinal bleeding risk factors (Figure 2). Similar percentages of women and men were diagnosed with renal disease within each database. In each database, a higher percentage of individuals with a musculoskeletal diagnosis had a CMCOI compared to the total study population (Figure 3).

\section{Discussion}

Evaluation of individuals in the PI, Medicaid, and MedicareSupplement claim databases showed that $\geq 50 \%$ with a PCP visit had one or more of the selected NSAID-relevant CMCOI (ie, asthma, cardiovascular risk factors, gastrointestinal bleeding risk factors, or renal impairment). We found that this percentage increased with age and that the percentage was universally higher among individuals who had a musculoskeletal diagnosis. The analysis also showed that women were as likely as men to have a nonaspirin, NSAID-relevant CMCOI. These healthrelated conditions need to be considered when consumers choose or HCPs recommend an OTC analgesic to adults of all ages.

Estimates of relative risk of NSAID-related asthma, cardiovascular risk, gastrointestinal bleeding, and renal impairment events varied depending on dose, duration, and source of exposure information (eg, survey of frequency of use, electronic medical records of dispensing), study design (eg, observational, randomized clinical), indication, and comparator (eg, no use, placebo, aspirin/acetaminophen). Following are some examples of cited relative risks. Data from meta-analyses and systematic reviews identified that
NSAID-related increases in relative risks for cardiovascular disease ranged from 1.22 to $2.26^{9-12}$ and for gastrointestinal bleeding ranged from 1.09 to 18.45 compared to placebo or no use or remote use of NSAIDs. ${ }^{11,13}$ Considering these relative risks along with the frequency of patient visits to an HCP for musculoskeletal and connective tissue diseases, as well as the frequency of one or more CMCOI among individuals in each database evaluated in this study, the potential magnitude of patients exposed to these NSAID-related risks is substantial.

Given the high proportion of patients diagnosed with one or more nonaspirin NSAID-relevant CMCOI and the high frequency of discussions about medicines that occur during a visit with an HCP, ${ }^{1}$ it is vital that patients inform their HCPs and that HCPs inquire about any changes in health status, as well as all medicines, OTC and prescription drugs, and supplements patients are taking. Research has addressed patient beliefs, perceptions, and behaviors related to OTC and prescription medicines. ${ }^{14-16}$ Counseling and education provided by community pharmacists improved patients' knowledge about nonaspirin NSAID-related safety issues. ${ }^{17}$ These favorable outcomes support the notion that primary HCPs can effectively educate patients about information available on OTC Drug Facts labels (eg, dosing, risks, warnings) and how that information supports why a specific analgesic is recommended, while a different analgesic is not. Moreover, HCPs should educate patients to understand that they should not assume that choice or dose of analgesics previously recommended to them is still acceptable. This is 
especially important, since individuals may not realize that an OTC pain reliever they have used in the past may no longer be the most appropriate choice for them, based on their changing age, coexisting medical conditions, and current medications. Of specific concern are patients with asthma and cardiovascular, gastrointestinal, and renal disorders, and older adults with several chronic conditions, since they are more likely than those without these disorders to experience adverse events while taking nonaspirin NSAIDs. ${ }^{6,18-21}$

Such patient-management challenges as these are addressed by guidelines published by professional societies and regulatory agencies, both of which recommend that HCPs prescribe or recommend the lowest dose of NSAID for the shortest duration to reduce the risk of complications..$^{3,6,18,19,22-31}$ Moreover, information available on the FDA website that is directed to HCPs reminds HCPs of the increased risks of serious cardiovascular events $(10 \%-50 \%)$ associated with NSAID use, regardless of an individual's history of or risk factors for cardiovascular disease. ${ }^{32}$

While HCPs are able to prescribe higher dosages of NSAIDs for defined durations while patients are under their care, HCPs should instruct their patients always to read OTC-product Drug Facts labels and follow dosage directions for both single dose and maximum daily dose when self-medicating with OTC NSAIDs. Health authorities and companies are continuously monitoring a medicine's safety profile and updating Drug Facts labels and patient-information leaflets accordingly. For example, updated warnings that strengthen and expand existing warnings relating to the risk of heart attack and stroke associated with NSAIDs were implemented in 2017 for all nonaspirin OTC NSAIDs. These include the addition of a heart attack and stroke warning, a warning to ask a doctor before use if you have had a stroke, and an updated direction to stop use and ask a doctor if you have symptoms of heart problems or stroke. HCPs should educate their patients to understand that OTC Drug Facts labels are not guidelines that are open to personal judgment, but rather specific and important instructions about dosing, duration of use, risks, and warnings meant to direct their safe and effective use of the product. Furthermore, HCPs should alert patients to Drug Facts label information, which instructs when they should contact their HCP (eg, before using if they have specific disorders, including ulcers, bleeding problems, high blood pressure, heart or kidney disease, or are 60 years of age or older $)^{33}$ and reinforce to their patients the importance of consulting with an HCP whenever they have additional questions. Communication and engagement with older patients with multiple chronic conditions or with their caregivers is vital while assessing treatment options, considering the increased likelihood of complexity related to balancing risks associated with treatment of chronic conditions with patients' preferences and priorities later in life. ${ }^{18}$

The current study confirms data collected by the National Ambulatory Medical Care Survey: the percentage of individuals reporting a diagnosis of any of the coexisting medical conditions increased with age and the percentage of individuals reporting musculoskeletal complaints increased with age until 65 years and then effectively stabilized. Furthermore, the current study defines the high frequency of nonaspirin NSAID-relevant CMCOI among patients with a musculoskeletal diagnosis as $>60 \%$.

In recognition of the fact that data used in the current analyses were extracted from health-care claim databases, some factors should be considered. The pool of patients in each database population was identified by a health-care claim for a visit to their primary HCP within the specified time frame. For the overall population, these visits may have been well-patient visits or visits due to a symptom including a musculoskeletal complaint or other reasons. Chronicity (ie, duration) was not determined or categorized for further analysis. Moreover, it was not possible to distinguish between acute and chronic status for any of the diagnoses (eg, musculoskeletal or cardiovascular disease). Records of filled prescriptions for medications to individuals were identifiable within each database; however, actual use of any medication by an individual could not be confirmed in the databases. It is important to note that the Medicare-Supplement population, which is covered by employer-sponsored private insurance, is likely to be healthier than the nonworking Medicare population.

Another potential limitation of this study was that patients with a diagnosis of a CMCOI and with frequent HCP follow-up visits may be more likely to be diagnosed with a musculoskeletal diagnosis during follow-up visits, simply because they have more opportunities to report musculoskeletal complaints. These individuals may naturally have more conditions diagnosed because they are already seeing an HCP for another condition. Nonetheless, the focus of the current study was to capture the clinical setting of an outpatient general practice (ie, family, internal medicine, or geriatric medicine practice) and specific disease or event risks the HCP might identify in a patient with or without a musculoskeletal diagnosis. It is important to recognize that within this setting, diagnoses and treatment recommendations could be based on any information available at the time, including the patient's medical record. Because we were interested in capturing risk information accessible to clinicians, our diagnoses were based on what appeared in the data, ie, the patient's medical 
record. The diagnosis codes for the selected coexisting conditions of interest and musculoskeletal disorders were based on a disease vocabulary that is created and maintained with clinical input through the Unified Medical Language System. Moreover, musculoskeletal diagnoses were required to be made in an outpatient general practice setting.

A protocol with defined risks and systematic methods for analysis was specified before the health-care claim databases were explored. That analysis plan included the decision not to conduct statistical analyses, since statistical analyses of such large databases could identify small mathematical differences that would qualify as statistically significant without necessarily being clinically relevant. It should be noted that the use of health-care claim databases served as a strength of the study. Each health-care claim database was representative of its respective population and provided a cross-sectional view of the US primary HCP population. Interestingly, findings were fairly consistent across databases and so likely represent primary HCP populations on a national basis.

\section{Conclusion}

These data show the prevalence of individuals who have CMCOI and the elevated prevalence of coexisting medical conditions among those presenting with a musculoskeletal disorder, as well as among older patients. The analyses reinforce the critical role HCPs can play in identifying patients with nonaspirin NSAID-relevant CMCOI, providing those patients with ongoing guidance on appropriate choice and use of OTC analgesics, and educating patients about the impact that aging, health status, concomitant conditions, and medicines has on selection of all medicines, including analgesics. Non-NSAID OTC analgesics and nonpharmacological treatment modalities may provide important alternatives for such patients.

\section{Abbreviations}

CMCOI, coexisting medical conditions of interest; FDA, US Food and Drug Administration; HCP, health-care professional; HIPAA, Health Insurance Portability and Accountability Act; IRB, institutional review board; MSD, musculoskeletal diagnosis; NDA, new drug application; NEIRB, New England Institutional Review Board; OTC, over the counter; PCP, primary-care professional; PI, Privately Insured; SNOMED, Systematized Nomenclature of Medicine.

\section{Data sharing statement}

All diagnosis and drug-code lists used in this study are available from the authors on reasonable request.

\section{Acknowledgments}

The authors thank Jesse A Berlin of Johnson and Johnson and Erica A Voss Stanochof Janssen Research and Development for their input and support in the development of this project. Johnson \& Johnson Consumer, McNeil Consumer Healthcare Division, provided funding for the study and for support provided to KE Boyle Consultants, LLC for this study (KEB).

\section{Author contributions}

LB, AEM, CB, and RW were responsible for the protocol/ study design, data review and analysis, critical review and revision of manuscript, final approval of the version to be published, agreement to be accountable for all aspects of the work, and questions being appropriately investigated and resolved; KEB was responsibile for drafting the manuscript, data review and analysis, critical review and revision of the manuscript, final approval of the version to be published, agreement to be accountable for all aspects of the work and questions being appropriately investigated and resolved.

\section{Disclosure}

LB and AEM are employees of Johnson \& Johnson Consumer Inc, McNeil Consumer Healthcare Division. RW and $\mathrm{CB}$ are employees of Janssen Research and Development. KEB is a consultant to Johnson \& Johnson Consumer Inc, McNeil Consumer Healthcare Division. The authors report no other conflicts of interest in this work.

\section{References}

1. Rui P, Okeyode T. National Ambulatory Medical Survey: 2015 state and national summary tables. Available from: https://www.cdc.gov/nchs/ data/ahcd/namcs_summary/2015_namcs_web_tables.pdf. Accessed January 14, 2019.

2. CDC. National Center for Health Statistics. Summary health statistics: NHIS; 2016. Available from: https://ftp.cdc.gov/pub/Health_Statistics/ NCHS/NHIS/SHS/2016_SHS_Table_A-4.pdf. Accessed January 14, 2019.

3. Hochberg MC, Altman RD, April KT, et al. American College of Rheumatology 2012 recommendations for the use of nonpharmacologic and pharmacologic therapies in osteoarthritis of the hand, hip, and knee. Arthritis Care Res. 2012;64(4):465-474.

4. Mcalindon TE, Bannuru RR, Sullivan MC, et al. OARSI guidelines for the non-surgical management of knee osteoarthritis. Osteoarthritis Cartilage. 2014;22(3):363-388.

5. Katz JN, Smith SR, Collins JE, et al. Cost-effectiveness of nonsteroidal anti-inflammatory drugs and opioids in the treatment of knee osteoarthritis in older patients with multiple comorbidities. Osteoarthritis Cartilage. 2016;24(3):409-418.

6. By the American Geriatrics Society 2015 Beers Criteria Update Expert Panel. American Geriatrics Society 2015 updated Beers criteria for potentially inappropriate medication use in older adults. $J$ Am Geriatr Soc. 2015;63(11):2227-2246. 
7. United States Department of Health and Human Services. Food and Drug Administration. Organ-specific warnings; internal analgesic, antipyretic, and antirheumatic drug products for over-the-counter human use; final monograph; technical amendment. 11/25/2009. Available from: https://www.federalregister.gov/documents/2009/11/25/ E9-28296/organ-specific-warnings-internal-analgesic-antipyretic-andantirheumatic-drug-products-for. Accessed January 13, 2019.

8. Scaglione S, Kliethermes S, Cao G, et al. The epidemiology of cirrhosis in the United States: a population-based study. J Clin Gastroenterol. 2015; 49(8):690-696.

9. Bally M, Dendukuri N, Rich B, et al. Risk of acute myocardial infarction with NSAIDs in real world use: Bayesian meta-analysis of individual patient data. BMJ. 2017;357:j1909.

10. McGettigan P, Henry D. Cardiovascular risk with non-steroidal antiinflammatory drugs: systematic review of population-based controlled observational studies. PLoS Med. 2011;8(9):e1001098.

11. Coxib and traditional NSAID Trialists' (CNT) Collaboration; Bhala N, Emberson J, et al. Vascular and upper gastrointestinal effects of non-steroidal anti-inflammatory drugs: meta-analyses of individual participant data from randomised trials. Lancet. 2013;382(9894): 769-779.

12. Trelle S, Reichenbach S, Wandel S, et al. Cardiovascular safety of non-steroidal anti-inflammatory drugs: Network meta-analysis. BMJ. 2011;342(1):c7086.

13. Castellsague J, Riera-Guardia N, Calingaert B, et al. On behalf of the Investigators of the safety of non-steroidal anti-inflammatory drugs (SOS) project. Drug Safety. 2012;35(12):1127-1146.

14. Wilcox CM, Cryer B, Triadafilopoulos G. Patterns of use and public perception of OTC pain relievers: focus on NSAIDs. J Rheumatol. 2005;32(11):2218-2224.

15. Hanna LA, Hughes CM. Public's views on making decisions about over-the-counter medication and their attitudes towards evidence of effectiveness: a cross-sectional questionnaire study. Patient Educ Couns. 2011;83(3):345-351

16. Boudreau DM, Wirtz H, von Korff M, Catz SL, St John J, Stang PE. A survey of adult awareness and use of medicine containing acetaminophen. Pharmacoepidemiol Drug Saf. 2013;22(3):229-240.

17. Jang SM, Cerulli J, Grabe DW, et al. NSAID-avoidance education in community pharmacies for patients at high risk for acute kidney injury, upstate New York, 2011. Prev Chronic Dis. 2014;11(11):E220.

18. Henrich WL, Agodoa LE, Barrett B, et al. Analgesics and the kidney: summary and recommendations to the Scientific Advisory Board of the National Kidney Foundation from an ad hoc committee of the National Kidney Foundation. Am J Kidney Dis. 1996;27(1):162-165.

19. Matthews ML. The role of dose reduction with NSAID use. Am J Manag Care. 2013;19(14 Suppl):S273-S277.

20. Brune K, Patrignani P. New insights into the use of currently available non-steroidal anti-inflammatory drugs. J Pain Res. 2015;8:105-118.
21. Pai AB, Divine H, Marciniak M, et al. National Kidney Disease Education Program Pharmacy Working Group. Need for a judicious use of nonsteroidal anti-inflammatory drugs to avoid community-acquired acute kidney injury. Ann Pharmacother. 2018;1:1060028018789174.

22. Meara AS, Simon LS. Advice from professional societies: appropriate use of NSAIDs. Pain Med. 2013;14(supp1 1):S3-S10.

23. American Geriatrics Society Expert Panel on the Care of Older Adults with Multimorbidity. Guiding principles for the care of older adults with multimorbidity: an approach for clinicians. J Am Geriatr Soc. 2012; 60(10):E1-E25.

24. Lanza FL, Chan FK, Quigley EM; Practice Parameters Committee of the American College of Gastroenterology. Guidelines for prevention of NSAID-related ulcer complications. Am J Gastroenterol. 2009; 104(3):728-738.

25. Taylor R, Lemtouni S, Weiss K, Pergolizzi JV. Pain management in the elderly: an FDA Safe Use Initiative Expert Panel's view on preventable harm associated with NSAID therapy. Curr Gerontol Geriatr Res. 2012;2012(7):1-9.

26. American Geriatrics Society Panel on Pharmacological Management of Persistent Pain in Older Persons. Pharmacological management of persistent pain in older persons. J Am Geriatr Soc. 2009;57(8):1331-1346.

27. Zhang W, Doherty M, Arden N, et al. EULAR Recommendations 2003: an evidence based approach to the management of knee osteoarthritis: Report of a Task Force of the Standing Committee for International Clinical Studies Including Therapeutic Trials (ESCISIT). Ann Rheum Dis. 2005; 64(5):669-681.

28. Jordan KM, Arden NK, Doherty M, et al. EULAR 2003 evidence based recommendations for the management of knee osteoarthritis ESCISIT. Ann Rheum Dis. 2003;62:1145-1155.

29. Zhang W, Nuki G, Moskowitz RW, et al. OARSI recommendations for the management of hip and knee osteoarthritis: Part III: changes in evidence following systematic cumulative update of research published through January 2009. Osteoarthritis Cartilage. 2010;18(4):476-499.

30. Richette P, Doherty M, Pascual E, et al. 2016 updated EULAR evidencebased recommendations for the management of gout. Ann Rheum Dis. 2017;76(1):29-42.

31. Khanna D, Khanna PP, Fitzgerald JD, et al. 2012 American College of Rheumatology guidelines for management of gout. Part 2: therapy and antiinflammatory prophylaxis of acute gouty arthritis. Arthritis Care Res. 2012;64(10):1447-1461.

32. FDA. Drug Safety and Availability. FDA Drug Safety Communication: FDA strengthens warning that non-aspirin nonsteroidal anti-inflammatory drugs (NSAIDs) can cause heart attacks or strokes. https://www.fda.gov/ Drugs/DrugSafety/ucm451800.htm. Accessed January 14, 2019.

33. FDA U.S. Postmarket drug safety information for patients and providers. Ibuprofen Drug Facts label. Available from: https://www.fda.gov/ Drugs/DrugSafety/PostmarketDrugSafetyInformationforPatientsandProviders/ucm125225.htm. Accessed January 14, 2019. 


\section{Supplementary material}

The Truven Health MarketScan ${ }^{\circledR}$ Commercial Claims and Encounters database (privately insured; [PI]) database is representative of a PI population of $>121$ million Americans from $\sim 350$ companies/employers in a wide range of industries and occupations in the US. The Truven Health MarketScan Multi-State Medicaid (Medicaid) database contains data on $>17$ million Medicaid enrollees from 12 contributing states. This database contains a blinded subset of Medicaid data derived from the pooled health-care experience of $\sim 6$ million Medicaid enrollees that is made available to private companies. This database represents $\sim 12$ million Medicaid beneficiaries each year. The Truven MarketScan Medicare Supplement (Medicare-Supplement) database is representative of Medicare-eligible active and retired employees and their Medicare-eligible dependents who are enrolled in employer-sponsored supplemental plans (predominantly fee-for-service plans). Medicare-Supplement represents $>9$ million PI Americans and a wide range of industries and occupations in the US. Only plans where both the Medicare-paid amounts and the employer-paid amounts were available and evident on the claims were selected for this database.

Each database includes inpatient and outpatient services, pharmacy claims, and health-plan enrollment data for all beneficiaries for services provided. Medical claim-capture information on inpatient and outpatient care includes date, type, and place of service, provider type, and payment information. ICD9-CM diagnosis codes, ICD9-CM procedure codes, and Current Procedural Terminology codes are provided for each medical claim. Pharmacy claim files capture the National Drug Code (NDC), dispensing date, quantity of drug, number of days supplied, and payment information.

Enrollment/eligibility records contain enrollment information and demographics (ie, year of birth, sex). For the Medicare-Supplement database, only individuals with both an employer-sponsored plan and Medicare benefits are included, indicating a high-cost health-care benefits plan and higher socioeconomic status. To allow for ease of use, data from these sources were converted to the Observational Medical Outcomes Partnership (OMOP) Common Data Model (CDM), version 5 (OMOP CDM) (Observational Health Sciences and Informatics Example ETLs) before any analyses were performed. The conversion of the databases to the OMOP CDM included the mapping of ICD-9-CM and NDC codes to Systematized Nomenclature of Medicine (SNOMED) and RxNorm standard concepts, respectively. To construct diagnosis definitions in the databases, one or more standard SNOMED concepts (OMOP Vocabulary version 5.0, April 3, 2015) was used for each of the four conditions/ risk groups of interest, and all terms from the lists were reviewed to exclude terms that were not consistent with the concept of interest. For example, for gastrointestinal bleeding risk, all descendant terms (using the SNOMED hierarchy) of the SNOMED concepts for gastrointestinal hemorrhage and gastrointestinal ulcer were used. In addition, gastrointestinal bleeding risk also includes all drugs linked to the RxNorm concepts for anticoagulants and corticosteroids. For cardiovascular risk, all descendant terms of the SNOMED concepts for cerebrovascular disease, heart failure, disorder of coronary artery, acute myocardial infarction, and hypertensive disorder were used.
Therapeutics and Clinical Risk Management

\section{Publish your work in this journal}

Therapeutics and Clinical Risk Management is an international, peerreviewed journal of clinical therapeutics and risk management, focusing on concise rapid reporting of clinical studies in all therapeutic areas, outcomes, safety, and programs for the effective, safe, and sustained use of medicines. This journal is indexed on PubMed Central, CAS,

\section{Dovepress}

EMBase, Scopus and the Elsevier Bibliographic databases. The manuscript management system is completely online and includes a very quick and fair peer-review system, which is all easy to use. Visit http://www.dovepress.com/testimonials.php to read real quotes from published authors. 